\title{
Calculations of Compound Nucleus Spin-Parity Distributions Populated via the $(p, t)$ Reaction in Support of Surrogate Neutron Capture Measurements
}

\author{
J.Benstead ${ }^{1,2, a}$, J.A.Tostevin ${ }^{2}$, J.E.Escher ${ }^{3}$, J.T.Burke ${ }^{3}$, R.O.Hughes ${ }^{3}$, S.Ota ${ }^{3}$, R.J.Casperson ${ }^{3}$, \\ and I.J.Thompson ${ }^{2,3}$ \\ ${ }^{1}$ AWE Aldermaston, Reading, Berkshire, RG7 4PR, United Kingdom \\ ${ }^{2}$ University of Surrey, Guildford, Surrey, GU2 7XH, United Kingdom \\ ${ }^{3}$ Lawrence Livermore National Laboratory, Livermore, California 94550, USA
}

\begin{abstract}
The surrogate reaction method may be used to determine the cross section for neutron induced reactions not accessible through standard experimental techniques. This is achieved by creating the same compound nucleus as would be expected in the desired reaction, but through a different incident channel, generally a direct transfer reaction. So far, the surrogate technique has been applied with reasonable success to determine the fission cross section for a number of actinides, but has been less successful when applied to other reactions, e.g. neutron capture, due to a 'spin-parity mismatch'. This mismatch, between the spin and parity distributions of the excited levels of the compound nucleus populated in the desired and surrogate channels, leads to differing decay probabilities and hence reduces the validity of using the surrogate method to infer the cross section in the desired channel. A greater theoretical understanding of the expected distribution of levels excited in both the desired and surrogate channels is therefore required in order to attempt to address this mismatch and allow the method to be utilised with greater confidence. Two neutron transfer reactions, e.g. $(\mathrm{p}, \mathrm{t})$, which allow the technique to be utilised for isotopes further removed from the line of stability, are the subject of this study. Results are presented for the calculated distribution of compound nucleus states populated in ${ }^{90} \mathrm{Zr}$, via the ${ }^{92} \mathrm{Zr}(\mathrm{p}, \mathrm{t})^{90} \mathrm{Zr}$ reaction, and are compared against measured data at an incident proton energy of $28.56 \mathrm{MeV}$.
\end{abstract}

\section{Introduction}

The majority of reactions of interest to the nuclear industry, and many relevant to astrophysics, involve the collision of an incident neutron with a target nucleus. Aside from elastic scattering, the reactions which may occur due to an incident neutron typically take place through an intermediate compound nucleus state, as shown in Figure 1.

Here the incident neutron is absorbed by, and shares its energy with, the target nucleus and forms an excited compound nucleus. This compound nucleus is unstable and will decay after some time to form the final products of the reaction. The basis of the surrogate reaction method is to exploit

\footnotetext{
ae-mail: james.benstead@awe.co.uk
} 


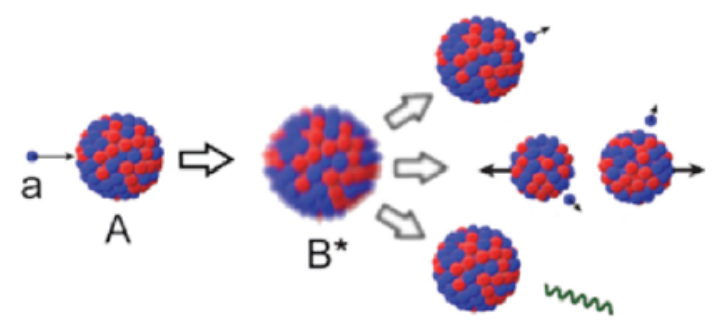

Figure 1. Illustration of a nuclear reaction proceeding through the compound nucleus stage. Here the projectile $a$ collides with $A$ to form $B^{*}$, which then decays to a number of possible products.

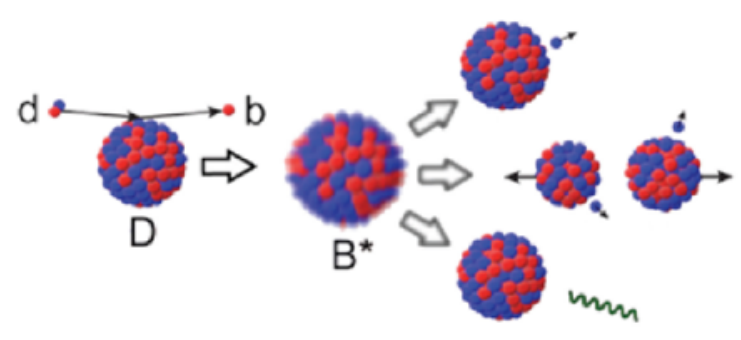

Figure 2. Illustration of the surrogate reaction method. Here, $d$ interacts with $D$ to form the compound nucleus $B^{*}$, which then decays in the same manner as would be expected through the desired reaction. The projectile $d$ may simply be scattered inelastically, but most often loses or gains nucleons to form particle $b$.

the Bohr assumption, that the mode of decay of a compound nucleus is independent of the type of reaction from which it formed [1]. It is assumed that only the spin distribution of the states, in both energy and angular momentum, populated in the compound nucleus plays a role in determining the statistical likelihood of decays via each possible channel [2, 3].

In the surrogate reaction method, a suitable surrogate nucleus and reaction are sought, such that the same compound nucleus, and if possible its spin distribution, will be formed as is expected in the reaction of interest. The surrogate process is illustrated in Figure 2.

In the desired reaction case, one needs to determine the cross section $\sigma_{\alpha \chi}\left(E_{a}\right)$ for a reaction with incident channel $\alpha(a+A)$ at an incident energy $E_{a}$ and exit channel $\chi$. The cross section $\sigma_{\alpha \chi}\left(E_{a}\right)$ is often split into two components,

$$
\sigma_{\alpha \chi}\left(E_{a}\right)=\sum_{J, \pi} \sigma_{\alpha}^{B^{*}}\left(E_{\mathrm{ex}}, J, \pi\right) G_{\chi}^{B^{*}}\left(E_{\mathrm{ex}}, J, \pi\right) .
$$

Here $\sigma_{\alpha}^{B^{*}}\left(E_{\mathrm{ex}}, J, \pi\right)$ is the cross section for $(a+A)$ forming the compound nucleus $B^{*}$ with an excitation energy $E_{\mathrm{ex}}$ in the state $J^{\pi}$ and $G_{\chi}^{B^{*}}\left(E_{\mathrm{ex}}, J, \pi\right)$ is the probability, or branching ratio, of $B^{*}$ decaying to channel $\chi$. 
In a standard measurement $\sigma_{\alpha \chi}\left(E_{a}\right)$ is measured directly. In the surrogate approach however, $G_{\chi}^{B^{*}}\left(E_{\mathrm{ex}}, J, \pi\right)$ is determined by indirect measurement and $\sigma_{\alpha}^{B^{*}}\left(E_{\mathrm{ex}}, J, \pi\right)$ is calculated, generally via the optical model. The surrogate approach is therefore intrinsically a hybrid of theory and experiment.

Early surrogate experiments employed the Weisskopf-Ewing approximation [4], which assumes that the probability of decay to any channel $\chi$ is independent of the $J^{\pi}$ components of the compound nucleus spin distribution, i.e. it is only dependent upon the excitation energy and $G_{\chi}^{B^{*}}\left(E_{\mathrm{ex}}, J, \pi\right) \rightarrow$ $G_{\chi}^{B^{*}}\left(E_{\mathrm{ex}}\right)$. The results of $(n, f)$ surrogate measurements generally compare favourably with direct experimental data [2]. However, generally, for each isotope studied, this match breaks down at lower excitation energies and also, in some cases, above the threshold for second-chance fission [2].

The poorer performance of the surrogate method in certain energy regions is due to a so-called spin mismatch and the breakdown of the validity of the Weisskopf-Ewing approximation [5]. At low excitation energies the available levels which a nucleus may occupy are discretely spaced in energy, with each level possessing a unique $J^{\pi}$ assignment. As the excitation energy increases, the density of levels increases until eventually a continuum of fragmented and overlapping states is reached. At lower excitation energies, a very particular spin distribution must be imparted to a target nucleus in order to populate the levels available with the same distribution strength as in the desired reaction channel. A surrogate reaction may quite easily populate a different spin distribution if the spin matching in the reaction used favours the transfer of very different values of $J^{\pi}$.

For fissile nuclei at higher excitation energies, the $(n, f)$ channel is generally dominant and the distribution of fragments generated, which may be altered by the differing fission-decay channels taken, is not important. Rather, it is the integrated $(n, f)$ cross section to all final products which is the value of concern. In other words, although the decay path taken from a higher excitation energy state may be different in the surrogate case compared to that of the desired reaction, it will still result in the production of fission fragments and hence the same $(n, f)$ cross section.

The limitations of the surrogate method, due to spin mismatch, are greater for the case of the $(n, \gamma)$ reaction. Surrogate $(n, \gamma)$ studies have shown that, for the current surrogate approach employed, a more sophisticated application of theory is required to take into account the differences in spin distribution between the desired and surrogate cases [6].

Another assumption made during early applications of the surrogate method was that differences in both the type and relative strength of pre-equilibrium reactions could be ignored in both the desired and surrogate reaction channels. Pre-equilibrium reactions are sensitive to the nature of the reaction entrance channel, given that they may include single-particle (or hole) excitations which depend upon the configuration of the orbitals of the nucleus. The configuration of orbitals is dependent upon whether the incident particle has been captured, as in the case of a neutron-induced reaction, or has scattered and removed nucleons, which could be the case in a direct reaction. The effect of differing pre-equilibrium reactions on the validity of the surrogate approach depends upon the specific reactions, both desired and surrogate, being investigated.

In parallel to the efforts to develop additional theory to 'translate' from a measured surrogate spin distribution to the optimal/expected one, new types of surrogate reactions which may populate spin distributions closer to that expected in the desired reaction are being investigated. Although early studies employed the $(t, p)$ reaction as a surrogate $[7,8],(p, t)$ has only been investigated for this purpose very recently $[9,10]$ and only in a preliminary manner.

In this study we have therefore developed a model for performing $(p, t)$ calculations over a range of excitation energies in order to predict the observables of a typical surrogate measurement. The model has been designed to require only very limited generic prior information regarding the target nucleus to which it is applied, with the expectation that it can be applied relatively quickly to a range of isotopes in order to better inform the selection of candidate surrogate experiments, i.e. those in which 


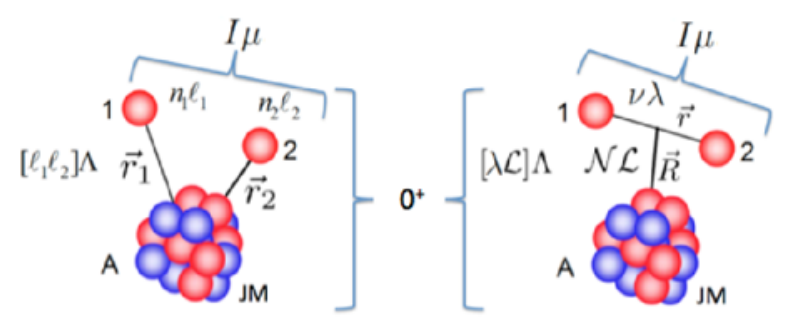

Figure 3. The various quantum numbers, angular momenta and their couplings present in the $(A+2)$-body target nucleus in the entrance channel in both the two neutron and di-neutron approaches.

the spin mismatch is minimised. This developed model has first been applied to recent measurements of the ${ }^{92} \mathrm{Zr}(p, t)^{90} \mathrm{Zr}$ reaction.

\section{Two-Neutron Transfer Model}

In this section we briefly describe the (p,t) model developed for this study. In order to simplify the development of the model we begin by studying specific, important cases. We make the following assumptions:

- The target nucleus is even-even, i.e. it possesses even numbers of both protons and neutrons, is spherical, and in its ground state has a spin and parity assignment of $J^{\pi}=0^{+}$.

- The two neutrons are transferred simultaneously, in one step, as a single spin-singlet 'di-neutron' object, with zero intrinsic angular momentum.

In order to perform calculations involving the transfer of a di-neutron, the quantum numbers and angular momenta of the two individual neutrons transferred must be translated into those of an equivalent single particle, as illustrated in Figure 3.

\subsection{Formalism}

Calculations of cross sections for the direct $(\mathrm{p}, \mathrm{t})$ transfer reaction on a mass $A+2$ target nucleus, populating specific $\mathrm{J}^{\pi}, A$-body final states, involve a number of components, see e.g. Glendenning [11]. The expression used for the cross section is

$$
\frac{d \sigma}{d \Omega}\left(0^{+} \rightarrow J^{\pi}\right)=\left|\sum_{N \Lambda S} G_{N \Lambda S J} B_{N \Lambda S J}\left(\vec{k}_{p}, \vec{k}_{t}\right)\right|^{2},
$$

where $B_{N \Lambda S J}$ is the (p,t) transition amplitude calculated via the Distorted Wave Born Approximation (DWBA) method and $G_{N \Lambda S J}$, comprised of nuclear structural factors, is broadly analogous to the spectroscopic amplitude of single-nucleon transfer reactions. More specifically, $G$ is given by the product of terms

$$
G_{\gamma N \Lambda S J}=\sum_{\gamma} g_{\gamma} \beta_{\gamma \Lambda S J} \Omega_{v}\left\langle v \lambda, N L ; \Lambda \mid n_{1} l_{1}, n_{2} l_{2} ; \Lambda\right\rangle .
$$

where $g_{\gamma}=\sqrt{2 /\left(1+\delta_{i j}\right)}$ is a symmetry factor dependent on the (like or unlike) pair of orbitals $i, j$ occupied by the two transferred neutrons, $\left\langle v \lambda, N L ; \Lambda \mid n_{1} l_{1}, n_{2} l_{2} ; \Lambda\right\rangle$ is a Moshinsky bracket [12], $\beta_{\gamma \Lambda S J}$ 
is the two-particle parentage coefficient, and $\Omega_{n}$ reflects the overlap of the two-nucleon relative motion wave functions between the initial and final states. $\Omega_{n}$ is assumed to be unity in the calculations presented here.

As we consider only even-even target nuclei and thus transfer from $0^{+}$ground states, the total and orbital angular momentum transferred are equal, $(\Lambda=) L=J$, and only natural parity final states with $\pi=(-1)^{J}$ are populated.

\subsection{Structural factors}

For the (p,t) pickup reaction the parentage coefficient, denoted by $\beta_{\gamma \Lambda S J}$, measures the component in the $\left(0^{+}\right)$mass $A+2$ target nucleus ground state of a specific $J^{\pi} A$-body residual nucleus final state plus two neutrons with quantum numbers $\gamma \equiv\left(\left[n_{1} l_{1} j_{1}\right],\left[n_{2} l_{2} j_{2}\right]\right)$ coupled to $[\Lambda, S] J$. Explicit forms relevant to our cases are detailed by e.g. Glendenning [11].

The value of the Moshinsky bracket gives the amplitude (within the harmonic oscillator approximation) for the overlap between the wave functions of the two neutron single-particle orbitals, i.e. $\gamma$, in the target nucleus and the $0^{+}$di-neutron configuration, $[L, S=0] J$. Here the $S=0$ and relative $\mathrm{s}$-wave restrictions are dictated, in the one-step approximation, by the $\langle\mathrm{p} \mid \mathrm{t}\rangle$ structure vertex with the outgoing triton.

\subsection{Energy levels}

The energies of the populated final states and the $Q$ values for the individual transitions required for the DWBA transfer calculations are a necessary input. For these we used the experimental twoneutron separation energy $S_{2 n}$ of the target nucleus combined with Hartree-Fock calculations of the energies of the bound neutron single-particle states. The different degenerate $J^{\pi}$ final states arising from the removal of neutrons from each pair of occupied neutron orbitals were split based on the phenomenological expectations, see e.g. Casten [13], from an attractive two-nucleon residual interaction.

The spherical Hartree-Fock [14] was used for these calculations. A variety of different Skyrme mean-field interactions were tested, but an inspection of previously identified levels, as shown in Figure 4, highlights a large number of levels with unassigned values of $J^{\pi}$, meaning determining a preferred potential is difficult. The SkX Skyrme model [15] was used for the current zirconium isotopes study.

\subsection{DWBA calculations}

The Surrey-version of the DWBA transfer code TwoFnR [16] was used for the (p,t) reaction cross section calculations. These cross sections showed little dependence on the choice of the proton optical model potential (OMP) selected. However, there was more significant sensitivity to the triton OMP used. Thus, calculations were performed using two available global triton OMPs $[17,18]$ to provide a first assessment of the uncertainty due to this physical input. In the results presented below, the proton OMP of Bechetti and Greenlees [19] was used with the triton OMP of Li et al. [17] unless otherwise stated.

\subsection{Final-state energy spreading}

There will be physical spreading, or fragmentation, of the strengths of the final states about their estimated energies (from the Hartree-Fock plus two-neutron residual interaction) [21]. We take this 


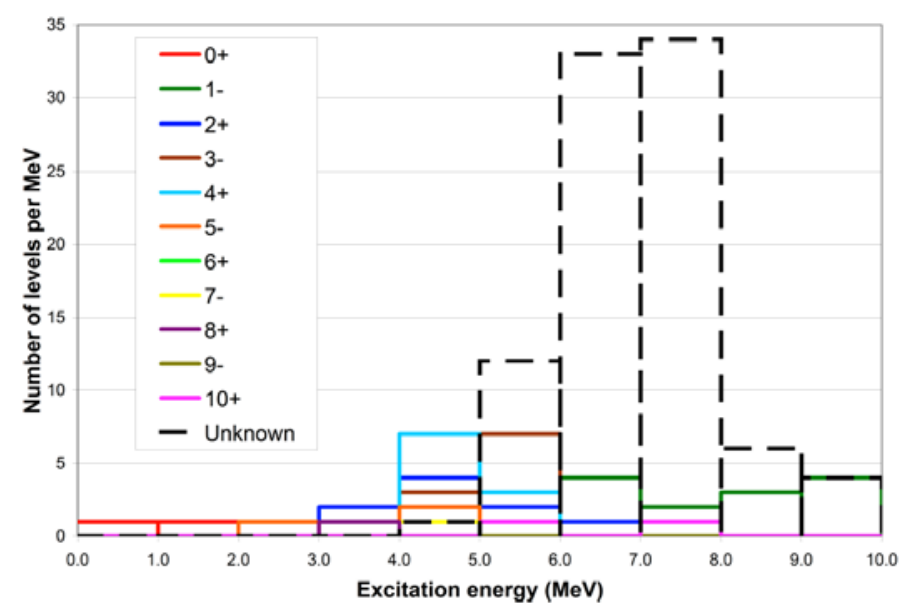

Figure 4. The density of levels of ${ }^{90} \mathrm{Zr}$ reported in the literature [20].

into account in the present calculations by the introduction of a parameterised spreading width, $\Gamma(E)$, for each state, dependent on its excitation energy above the Fermi energy of the residual nucleus $E_{F}$. We assume

$$
\Gamma(E)=\frac{1}{2}\left[\frac{\epsilon_{0}\left(E-E_{F}\right)^{2}}{\left(E-E_{F}\right)^{2}+E_{0}^{2}}+\frac{\epsilon_{1}\left(E-E_{F}\right)^{2}}{\left(E-E_{F}\right)^{2}+E_{1}^{2}}\right],
$$

where $\epsilon_{0}, \epsilon_{1}, E_{0}, E_{1}$ are chosen constants; taken here from Ref. [23] where they were used for ${ }^{60} \mathrm{Ni}$.

This approach, apart from an additional factor of $\frac{1}{2}$, follows that of Brown and Rho [22]. The spreading of the strength of the transfer yield with excitation energy is distributed with a Breit-Wigner shaped form factor with a FWHM of $\Gamma(E)$. This method has been employed for related analyses of yield distributions following single-nucleon transfer reactions. The width calculated from Equation 4 is approximately linear with excitation energy for excited states below $25 \mathrm{MeV}$, and has a width $\Gamma(E) \approx 7 \mathrm{MeV}$ at $\left(E-E_{F}\right)=25 \mathrm{MeV}$.

\section{Experimental Data}

The experimental data compared to in this study were recorded in mid-2013. A $28.56 \mathrm{MeV}$ proton beam was bombarded onto a thin $\approx 1 \mathrm{mg} / \mathrm{cm}^{2}$ isotopically purified ${ }^{92} \mathrm{Zr}$ foil. A large number of separate irradiations were conducted throughout the experiment with a total beam on target time of over 300,000 seconds (approximately 3.5 days). The average beam current was $1.56 \mathrm{nA}$.

\subsection{STARS}

The Silicon Telescope Array for Reaction Studies (STARS) is an annular array of (Micron Semiconductor S2) silicon detectors used for the detection and identification of charged particles emitted from nuclear reactions [24].

In a typical setup, as illustrated in Figure 5, the STARS detector is placed within a scattering chamber behind a foil target. This foil is then bombared by incident charged particles and STARS located 
in the downstream position will detect the charged particles emitted, generally via direct reactions, into a range of scattering angles. The typical angular coverage of STARS is not typical and generally $\theta=35^{\circ}$ to $55^{\circ}$. The STARS array is pixelated and can therefore record the angular distributions of emitted particles as well as their energies.

Measurements of the angular distribution of a state at a specific energy may be used to determine the $J^{\pi}$ of that state. Unfortunately, in the measurements, the point of beam/target interaction was offset from the central value, leading to coarser resolution angular distributions to be observed for individual states.

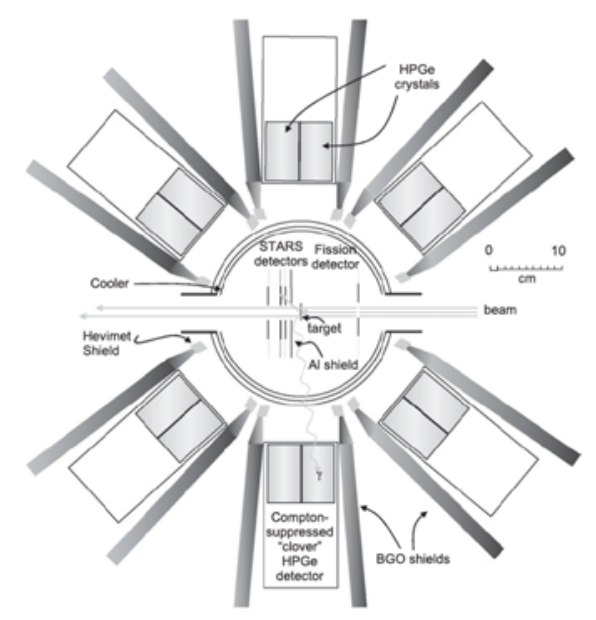

Figure 5. An overview of the STARLiTeR detector.

STARS is located at the K150 Superconducting Cyclotron Facility at Texas A\&M University. STARS is fielded with an array of HPGe detectors referred to as the Livermore Texas Richmond (LiTeR) array to form the STARLiTeR detector. The ability to detect $\gamma$-rays in coincidence with outgoing particle angular distributions allows for the identification of specific states populated in the excited residual nucleus and their decay pathways.

\section{$3.2(p, t)$ measurement}

The primary purpose of this ${ }^{92} \mathrm{Zr}$ foil irradiation was unfortunately not for studies of the ${ }^{92} \mathrm{Zr}(p, t){ }^{90} \mathrm{Zr}$ reaction. Rather the experiment was tailored to study the ${ }^{92} \mathrm{Zr}(p, d)^{91} \mathrm{Zr}$ reaction as a potential surrogate for the ${ }^{90} \mathrm{Zr}(n, \gamma){ }^{91} \mathrm{Zr}$ reaction. This meant that the incident proton energy was optimised for measurements of the $J^{\pi}$ distribution of ${ }^{91} \mathrm{Zr}$ at energies around its $S_{n}$ value. Fortunately, given the ability of STARLiTeR to differentiate between outgoing deuterons and tritons, data for the ${ }^{92} \mathrm{Zr}(p, t)^{90} \mathrm{Zr}$ reaction were also collected.

However, the energies of the states populated in ${ }^{90} \mathrm{Zr}$ are at energies below its $S_{n}$ value, meaning that these data are unsuitable for direct use in a ${ }^{89} \mathrm{Zr}(n, \gamma){ }^{90} \mathrm{Zr}$ surrogate study. 


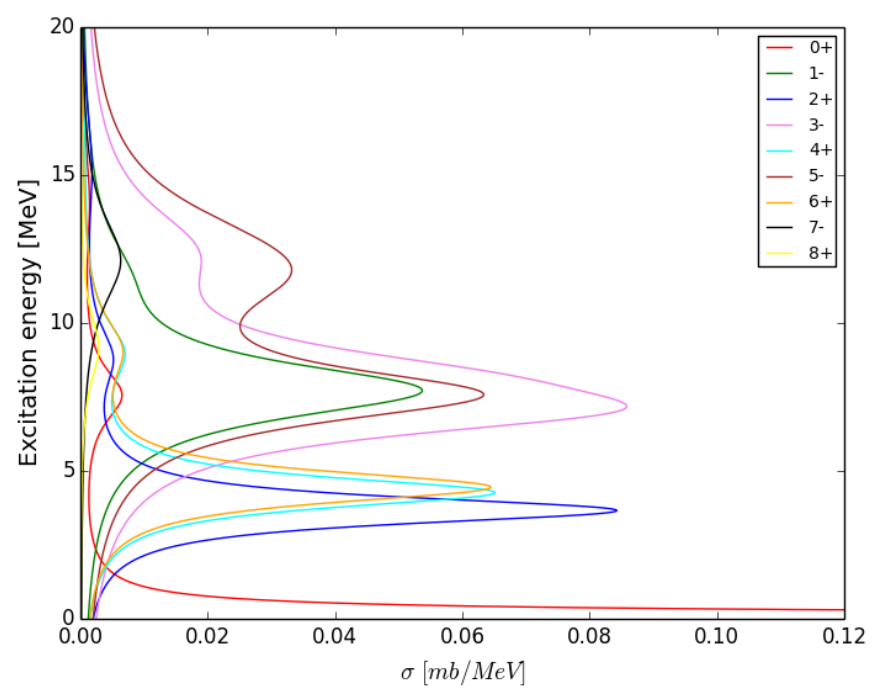

Figure 6. Calculated spin distribution for ${ }^{90} \mathrm{Zr}$ as a function of excitation energy following the ${ }^{92} \mathrm{Zr}(p, t)$ reaction.

\section{Results and Analysis}

\subsection{Spin distribution}

The model described above was used to calculate the $J^{\pi}$ distribution for the excited states in ${ }^{90} \mathrm{Zr}$ expected to be populated following the ${ }^{92} \mathrm{Zr}(p, t)^{90} \mathrm{Zr}$ reaction. This distribution is shown in Figure 6 , with the individual levels summed over each value of $J^{\pi}$. The states have been spread in energy according to the method of Brown and Rho outlined in Section 2.5.

The excitation energies of these states may be translated into the energies of the tritons which would be emitted following their excitation (and are actually observed in the experiment). Figure 7 shows the normalised and summed $J^{\pi}$ distribution as a function of outgoing triton, or rather product, kinetic energy, compared against the normalised measured data. The cross section is now only that for tritons incident on the inner radial ring of the STARS detector.

\subsection{Identification of states}

Figure 8 compares the calculated and measured data for the case of unspread levels (apart from spreading due to the experimental resolution). In this case, the comparison is for the triton distribution over the entire STARS detector. This is to avoid possible differences in the calculated positions of maxima and minima in angular distributions from masking the presence of a state. As may be seen in Figure 8 , there is a mismatch in the positions of the calculated levels with those measured. However, it was found that this match does not improve through the use of either a different Skyrme potential in the Hartree-Fock level energy calculations or a more sophisticated shell model calculation. Rather the differences in the number of levels present and/or their energies may be due to fragmentation [21] and hence accounted for in a general sense via our energy spreading prescription described previously. 


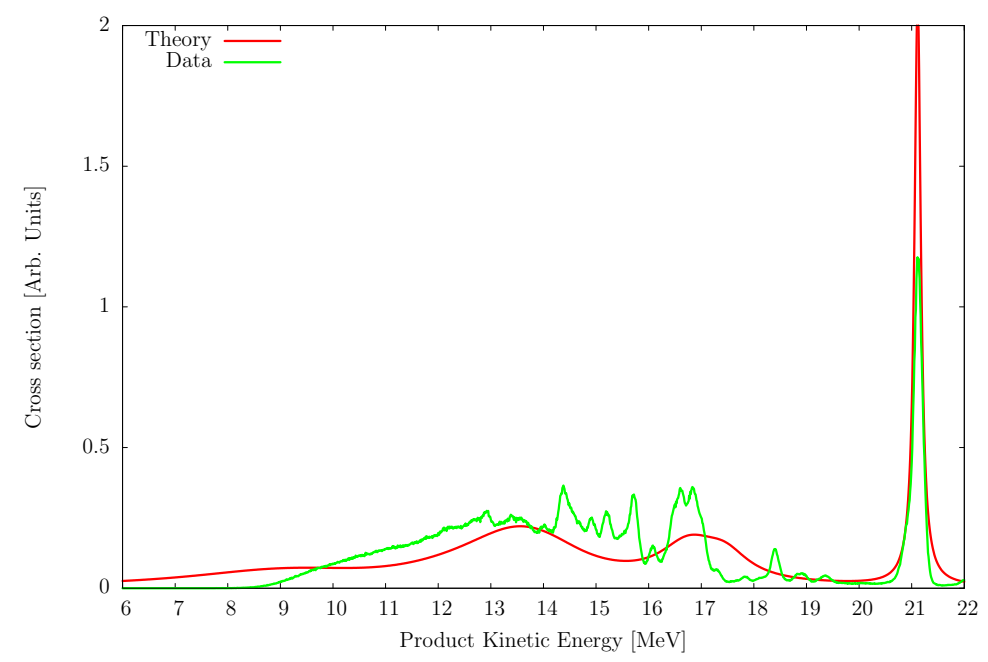

Figure 7. Comparison of theoretical results, using the half Brown and Rho spreading width, against experimental data for all rings of the detector.

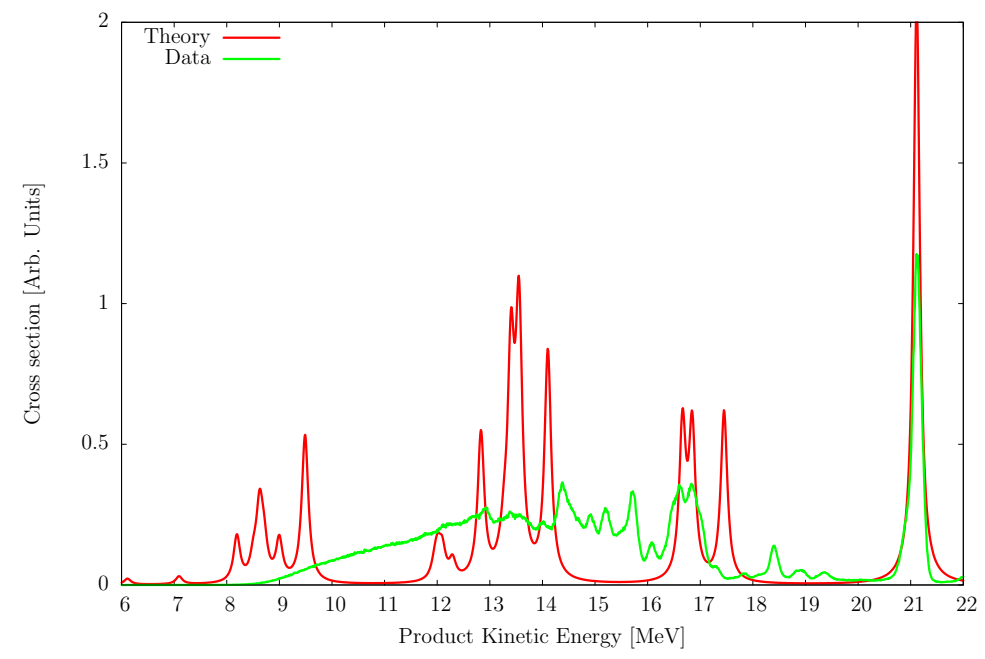

Figure 8. Theory vs data comparison for the triton spectrum integrated over all rings of the detector. In this case the theoretical states contributing to the total distribution are only spread by a width equal to that of the experimental resolution.

The angular distribution of tritons emitted due to the population of a specfic state will be related to its $J^{\pi}$ value. By comparing the calculated angular distribution of a state with that of a measured state, at a coincident energy, we are able to better determine if we are calculating actual physical states. 


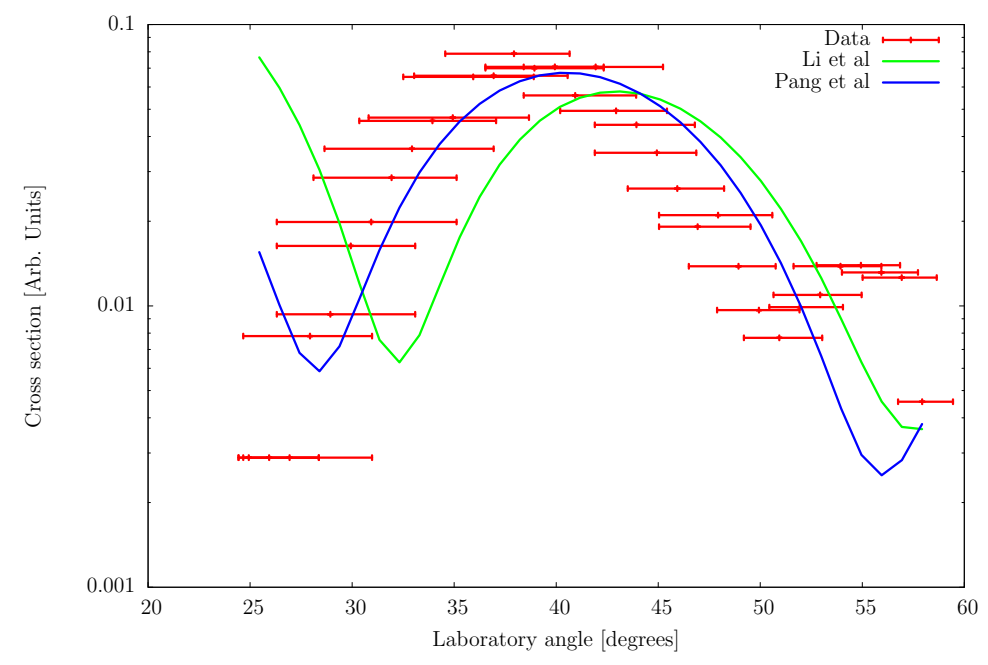

Figure 9. Comparison of the experimental angular distribution for the $0^{+}$ground state against the model predictions made using both the Li et al. and Pang et al. OMPs.

Figure 9 shows a comparison for the $0^{+}{ }^{90} \mathrm{Zr}$ ground state. It was found that the Li et. al. global triton OMP did not produce a good match against the experimental data. However, a calculation using the alternative Pang et. al. [18] OMP considered for this study produces a better match and perhaps indicates that further work to identify a preferential triton, and possibly proton, OMP should be performed.

\subsection{Contaminants}

It is possible that tritons may be observed which originate from $(p, t)$ reactions on the other $\mathrm{Zr}$ isotopes present as contaminants in the ${ }^{92} \mathrm{Zr}$ target. Tritons emitted from the direct population of the ${ }^{89} \mathrm{Zr}$ and ${ }^{88} \mathrm{Zr}$ ground states will fall into our energy range of interest. The peak corresponding to the ${ }^{89} \mathrm{Zr}$ ground state will be at $E_{t}^{\prime}=17.85 \mathrm{MeV}$ and that corresponding to the ${ }^{88} \mathrm{Zr}$ ground state at $E_{t}^{\prime}=15.72$ $\mathrm{MeV}$. An inspection of the data, combined with a knowledge of the estimated size of each peak relative to the ${ }^{90} \mathrm{Zr}$ ground state peak, suggests that the ${ }^{88} \mathrm{Zr}$ peak will be unobservable due to the background of tritons from the ${ }^{92} \mathrm{Zr}(p, t){ }^{90} \mathrm{Zr}$ reaction in that region. However, the peak due to ${ }^{89} \mathrm{Zr}$ is possibly visible in the data above background at $E_{t}^{\prime}=17.85 \mathrm{MeV}$.

\subsection{Unnatural parity states}

The model developed implicitly includes the assumption that unnatural-parity states are not populated. This is due to the two neutrons being transferred in a single step as a di-neutron, possessing an intrinsic spin of $S=0$. Despite this assumption, the model still calculates the energies and $J^{\pi}$ values of potential unnatural-parity states which would be populated via the transfer of various neutron pairs.

Table 1 lists the unnatural parity levels previously observed in ${ }^{90} \mathrm{Zr}$ [25], along with the two unassigned levels previously seen via the ${ }^{92} \mathrm{Zr}(p, t){ }^{90} \mathrm{Zr}$ reaction, and compares these against the energies 
Table 1. The excitation energies of unnatural-parity levels previously observed in ${ }^{90} \mathrm{Zr}$ [25]. Also shown are the energies of predicted potential unnatural-parity states along with the quantum numbers of the relevant transferred neutron pair.

\begin{tabular}{ccccc|cc}
\hline & Theory & & & Measured \\
$E^{*}[\mathrm{MeV}]$ & $J^{\pi}$ & $N$ & $n_{1} l_{1} j_{1}$ & $n_{2} l_{2} j_{2}$ & $E^{*}[\mathrm{MeV}]$ & $J^{\pi}$ \\
\hline & & & & & 4.238 & $?$ \\
& & & & & 4.540 & $6^{-}$ \\
4.613 & $3^{+}$ & 2 & $0 g_{9 / 2}$ & $1 d_{5 / 2}$ & & $1^{+}$ \\
4.613 & $5^{+}$ & 1 & $0 g_{9 / 2}$ & $1 d_{5 / 2}$ & & \\
& & & & & 5.060 & $7^{+}$ \\
& & & & & 5.068 & $?$ \\
7.010 & $2^{-}$ & & $1 p_{1 / 2}$ & $1 d_{5 / 2}$ & & \\
& & & & & 7.248 & $9^{+}$ \\
7.992 & $2^{-}$ & & $0 f_{5 / 2}$ & $1 d_{5 / 2}$ & & $2^{-}$ \\
7.992 & $4^{-}$ & & $0 f_{5 / 2}$ & $1 d_{5 / 2}$ & & \\
& & & & & 8.032 & $2^{-}$ \\
8.510 & $2^{-}$ & & $1 p_{3 / 2}$ & $1 d_{5 / 2}$ & & \\
\hline
\end{tabular}

of the calculated unnatural parity states. It should be noted that transitions to unnatural states are not split by a residual interaction.

As can be seen in Table 1, the two predicted unnatural parity states at $4.613 \mathrm{MeV}$ could be potential candidates for the unassigned levels at $4.238 \mathrm{MeV}$ and $5.068 \mathrm{MeV}$. The two observed 2- levels have predicted counterparts at energies which, although not the same, are close enough to provide a reasonable match given the limitations of our Hartree-Fock approach.

\section{Summary and Outlook}

A theoretical model has been developed to predict the excitation energy, spin and parity distributions of the residual nuclei following a $(p, t)$ two-neutron transfer reaction. This model has been designed to be purely predictive and to require little or no detailed prior information on the target nucleus in question. The model developed has been applied to the case of $28.56 \mathrm{MeV}$ protons incident on an isotopically enriched ${ }^{92} \mathrm{Zr}$ target, a case for which experimental data have recently been taken by a group from the Lawrence Livermore National Laboratory using the STARLiTeR detector at Texas A\&M University.

\subsection{Efficacy of the developed $(p, t)$ model}

The model developed makes a number of assumptions: the target nucleus is even-even, spherical; it is in its ground state with $J^{\pi}=0^{+}$; and that the two neutrons are transferred simultaneously, in one-step, as a single spin-singlet di-neutron object, with zero intrinsic angular momentum. A detailed comparison between the model and the data for ${ }^{92} \mathrm{Zr}(p, t)^{90} \mathrm{Zr}$ shows a reasonable match to the average trends of the observed spectra, but a breakdown when individual discrete states are scutinised in detail. In particular, the model fails to predict the population of a number of physical states observed in ${ }^{90} \mathrm{Zr}$, suggesting a more sophisticated approach to the structural and/or dynamical components of the model is required. 


\subsection{Suitability of ${ }^{92} \mathrm{Zr}(\boldsymbol{p}, \boldsymbol{t})$ as a surrogate for ${ }^{89} \mathrm{Zr}(\boldsymbol{n}, \gamma)$}

Unfortunately, as the ${ }^{92} \mathrm{Zr}(p, t)^{90} \mathrm{Zr}$ data used in this study were taken as part of an experiment tailored for $(p, d)$, the energy of the proton beam was too low for the triton spectrum, at energies corresponding to excitation energies in the residual nucleus $A$ at $\approx S_{n}^{A}$, to be observed. Therefore, the spin distribution calculated to be populated in this region cannot be compared to experimental data. Applying the model to a different $\mathrm{Zr}$ isotope for which experimental $(\mathrm{p}, \mathrm{t})$ data exist in the correct energy regime to act as an $(\mathrm{n}, \gamma)$ surrogate will be explored in a future study.

\subsection{Outlook}

The developed $(p, t)$ model is sufficiently mature that it could be applied, with only minimal effort to any even-even, spherical, $0^{+}$target nucleus. It is hoped that this tool may prove of some use to the surrogate reaction community in helping to predict spin distributions populated via $(p, t)$. However, it is acknowledged that an experiment resulting in a higher excitation energy of the residual compound nucleus is required in order to assess the model against data in the correct energy regime, i.e. at excitation energies around $S_{n}$.

\section{Acknowledgments}

This work was supported by the UK Ministry of Defence and was performed under the auspices of the US Department of Energy by Lawrence Livermore National Laboratory under contract DEAC52-07NA27344. JAT acknowledges support of the Science and Technology Facilities Council (UK) grants ST/J000051 and ST/L005743. S.O. is supported by a JSPS Postdoctoral Fellowship for Research Abroad. British Crown Owned Copyright (2015)/AWE.

\section{References}

[1] J.M. Blatt and V.F. Weisskopf, Theoretical nuclear physics (John Wiley \& Sons, 1952)

[2] J.E. Escher et. al., Reviews of Modern Physics 84, 353 (2012)

[3] G. Kessedjian et. al., Phys. Lett. B 692(5), 297 (2010)

[4] V.F. Weisskopf and D.H. Ewin, Phys. Rev 57(6), 472 (1940)

[5] J.E. Escher and F.S. Dietrich, Phys. Rev. C 81, 024612 (2010)

[6] N.D. Scielzo et. al., Phys. Rev. C 81, 034608 (2010)

[7] J.D. Cramer and H.C. Britt, Nucl. Sci. Eng. 41, 177 (1970)

[8] H.C. Britt and J.B. Wilhelmy, Nucl. Sci. Eng. 72, 2 (1979)

[9] R.O. Hughes et. al., Phys. Rev. C 85, 024613 (2012)

[10] R.O. Hughes et. al., Phys. Rev. C 90, 014304 (2014)

[11] N.K. Glendenning, Phys. Rev. B 137, 102 (1965)

[12] M. Moshinsky, Nucl. Phys. 13, 104 (1959)

[13] R.F. Casten, Nuclear structure from a simple perspective (Oxford University Press, second edition, 2000)

[14] B.A. Brown et. al., Code DENs, provided as part of oxBASH, Oxbash for Windows (MSU-NSCL report number 1289, 2004)

[15] B.A. Brown, Phys. Rev. C 58, 220 (1998)

[16] J.A. Tostevin, http://www.nucleartheory.net/NPG/code.htm (last accessed 6 October 2015) 
[17] X. Li et. al., Nucl. Phys. A 789, 103 (2007)

[18] D.Y. Pang et. al., Phys. Rev. C 79, 024615 (2009)

[19] F.D. Becchetti and G.W. Greenlees, Phys. Rev. 182(4), 1190 (1969)

[20] C.M. Baglin, Nuclear Data Sheets 113(10), 2187 (2012)

[21] M.G.E. Brand et. al., Nucl. Phys. A 531(2), 253 (1991)

[22] G.E Brown and M. Rho, Nucl. Phys. A 372(3), 397 (1981)

[23] M. Matoba et. al., Nucl. Phys. A 581, 21 (1995)

[24] S.R. Lesher et. al., Nucl. Instr. and Meth. in Phys. Res. Section A: Accelerators, Spectrometers, Detectors and Associated Equipment 621, 286 (2010)

[25] E. Browne, Nuclear Data Sheets 82(3), 379 (1997)

[26] A.J. Koning et. al., Proceedings of the International Conference on Nuclear Data for Science and Technology, April 22-27, 2007, Nice, France, pp211-214 